\title{
Protección al consumidor y la delegación de competencia de INDECOPI a la Superintendencia Nacional de Salud, en el marco del Decreto Legislativo No 1158 Protection to Consumer and INDECOPI Competence Delegation to the National Health Superintendence, under the Framework of the Legislative Decree 1158
}

Sara Ynés Tello Cabello* y Nicolaz Martín Dionicio Gutarra Perochena**

http://dx.doi.org/10.21503/lex.v14i17.944

* Abogada por la Universidad Inca Garcilaso de la Vega, doctora en Educación por la Universidad Alas Peruanas, máster en Derecho Empresarial por la Universidad de Lima, egresada del Doctorado en Derecho por la Universidad Nacional Mayor de San Marcos, Diploma de Especialización en el 13er Curso Anual en "Gestión y Dirección de Empresas" por la Pontificia Universidad Católica del Perú; consultora empresarial. Docente de la Facultad de Derecho y Ciencia Política de la UAP, y de la Facultad de Contabilidad y Finanzas de la Universidad San Martín de Porres en los cursos de Derecho Empresarial y Derecho Tributario. E-mail: s_tello@doc.uap.edu.pe

** Estudiante del XI ciclo de la carrera de Derecho de la Universidad Alas Peruanas, colaborador de la Gerencia Central de Seguros y Prestaciones Económicas de ESSALUD y asesor representante en el Centro MAC de la Presidencia de Consejo de Ministros, con experiencia en ponencias institucionales y académicas sobre Derecho Administrativo y Derecho de la Seguridad Social. Destacada participación en el Concurso Nacional de Ensayos en Derechos Fundamentales, edición 2015, del Ministerio de Justicia y Derechos Humanos. E-mail: nicolaz.gutarra@gmail.com

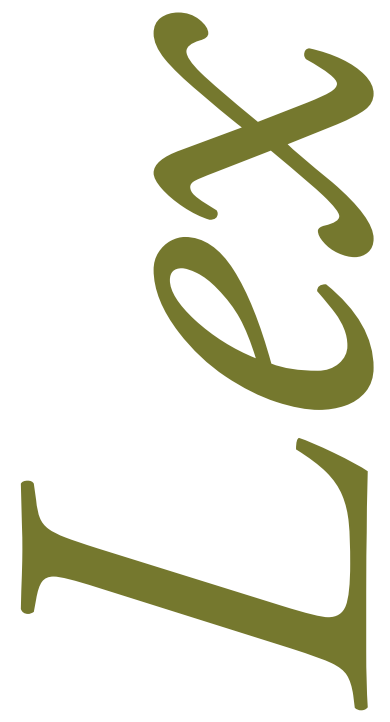




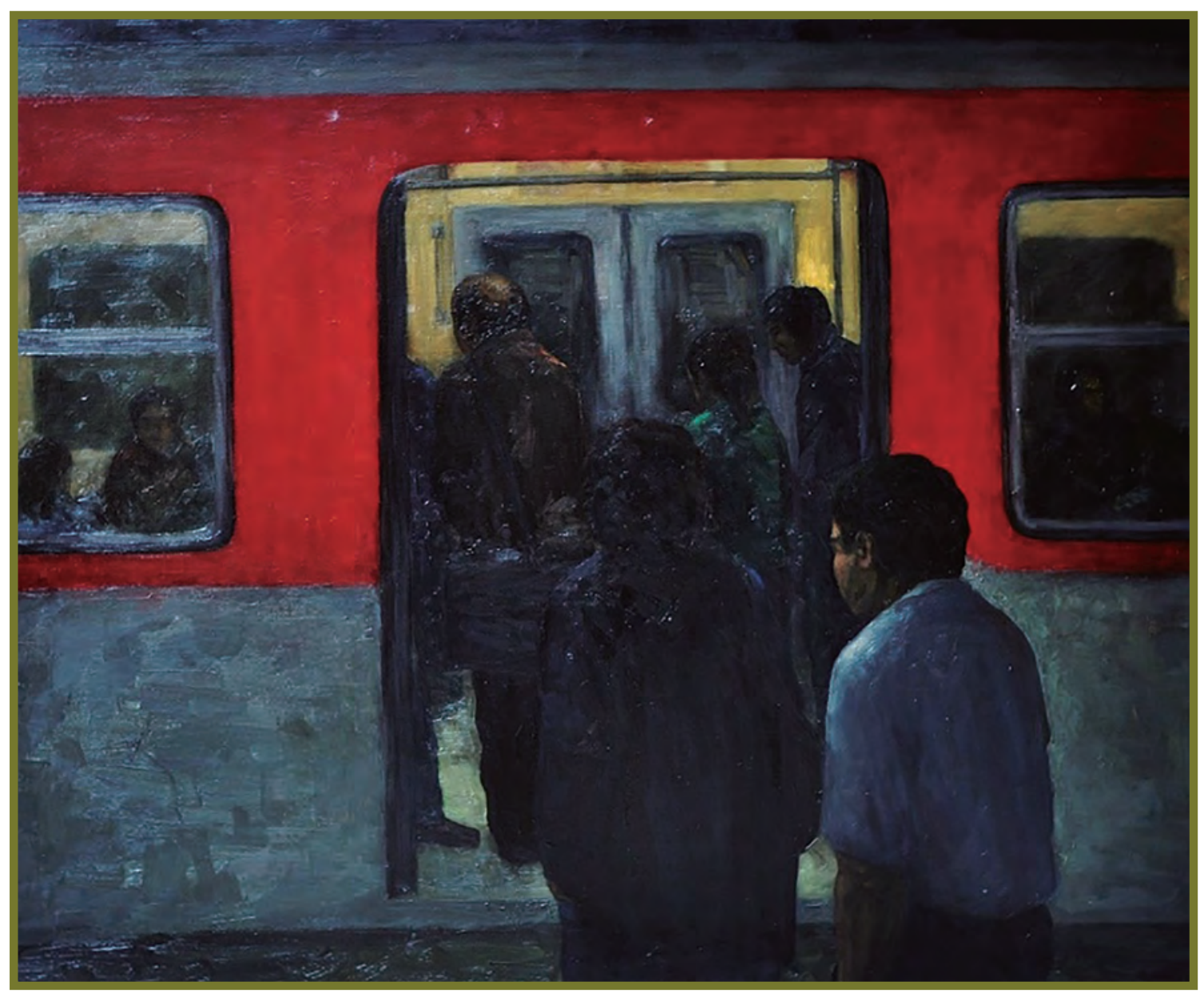

Metro $(81 \mathrm{~cm}$ x $100 \mathrm{~cm})$. Diego Alcalde Taboada. 


\section{RESUMEN}

El presente artículo tiene como objetivo el análisis normativo de la delegación de competencia de INDECOPI a la Superintendencia Nacional de Salud, en el marco del Decreto Legislativo $1158^{\circ}$, respecto a las Instituciones Prestadoras de Servicios de Salud y a las Instituciones Administradoras de Fondos de Aseguramiento.

Palabras clave: protección al consumidor, delegación de competencia de INDECOPI a la Superintendencia Nacional de Salud.

\section{ABSTRACT}

This article has as an aim a normative analysis of the INDECOPI Competence Delegation to the National Health Superintendence under the framework of the Legislative Decree 1158, in relation to the Health Care Facility Institutions and the Assurance Funds Management Institutions.

Key words: protection to the consumer, INDECOPI competence delegation to the National Health Superintendence. 


\section{INTRODUCCIÓN}

El Código de Protección y Defensa del consumidor ha despertado todo tipo de comentarios, y por supuesto no ha faltado la opinión de economistas del Derecho que lo critican como una muestra de socialismo, en virtud del cual, según ellos, el consumidor es tratado como un incapaz ya que el Estado decide lo que es mejor para el individuo, incluso sobre él mismo. Por lo tanto, la única solución que se propone es dejar que el consumidor en forma "libre" escoja dentro de la múltiple oferta que se produce gracias a la "competencia" de nuestro mercado. Se dice textualmente que "el poder radica en la libertad de elegir". ${ }^{1}$

Viendo ello, debemos mencionar que nuestro sistema de salud per se es complejo, puesto que lo conforman diversas entidades entre públicas y privadas y cada una clasificada por factores de financiamiento, servicios, planes y coberturas. Ante esta gama de agentes involucrados es imposible que no se generen conflictos entre los usuarios y los encargados de prestar servicios.

Debido a la gran cantidad de casos registrados y/o denunciados, era necesario canalizar ese reclamo legítimo frente a la vulneración de derechos. Diversas entidades se encargaban de resolver los conflictos generados entre entidades de servicios de salud y los usuarios; sin embargo, aun así se requería de un ente especializado que ayudase a morigerar las situaciones contraproducentes que muchos ciudadanos padecían.

Para el presente artículo, analizaremos el Título IV, Capítulo II del Código de Protección y Defensa del Consumidor, correspondiente a productos o servicios de salud; así mismo realizaremos un análisis exhaustivo de la delegación de competencia de INDECOPI a la Superintendencia Nacional de Salud, en el marco del Decreto Legislativo 1158; para ello transitamos por el Código de Protección y Defensa del Consumidor, y explicaremos de forma cronológica cómo fueron implementándose diversos entes involucrados en la protección de derechos de los usuarios de servicios de salud, al igual que sus competencias, limitantes, base legal y su evolución, hasta llegar a conformarse la actual Superintendencia Nacional en Salud.

Alfredo Bullar Gonzales, "Código de Consumo: el discreto encanto del socialismo", El Comercio, 10 de abril de 2010, A-6. 


\section{PRODUCTOS O SERVICIOS DE SALUD}

De acuerdo al artículo $7^{\circ}$ de la Constitución Política del Perú, todos tienen derecho a la protección de la salud. Siguiendo esa línea, la jurisprudencia del Tribunal Constitucional del Perú señala: "La persona humana por su dignidad tiene derechos naturales anteriores a la sociedad y al Estado, inmanentes a sí misma, los cuales han sido progresivamente reconocidos hasta hoy en la legislación positiva como derechos humanos de carácter universal, entre los cuales el derecho a la vida y a la salud, ligados al instinto de conservación, son de primerísimo orden e importancia, se hallan protegidos inclusive a través de tratados internacionales que obligan al Perú, porque en ellos se funde la legitimidad moral de toda autoridad". ${ }^{2}$

En el año 1993 se produce el cambio constitucional, reforzándose la protección a los consumidores, al establecer el artículo $65^{\circ}$ de la Constitución Política del Perú que "El estado defiende el interés de los consumidores y usuarios. Para tal efecto garantiza el derecho a la información sobre los bienes y servicios que se encuentren a su disposición en el mercado. Así mismo vela, en particular, por la salud y seguridad de la población”. Esta norma es muy importante, no solo porque establece un sólido anclaje constitucional para la protección constitucional, sino porque expresamente establece que el fundamento de esta es su derecho a estar adecuadamente informado sobre los bienes y servicios que adquiere, buscando corregir así las consecuencias negativas que puede producir la asimetría informativa ${ }^{3}$.

Es así que el Estado, en la búsqueda mínima de la protección al consumidor, ha delegado ciertas funciones en protección al consumidor a la Superintendencia Nacional de Salud, en el marco del Decreto Legislativo 1158. Teniendo en cuenta que la persona humana es el fin supremo de la sociedad y del Estado, requiere de una protección en la prestación de salud.

Es por ello que el Código de Protección y Defensa del Consumidor le dedica en su Título IV, Capítulo II, la protección a los productos o servicios de salud. La intención del legislador es la protección del consumidor en los establecimientos de salud, imperando para ello la defensa del principio de Igualdad ${ }^{4}$.

Miguel Ramos Bohórquez, Nuevo Código de Protección y Defensa del Consumidor. Ley N² 29571, edición $2014,98$.

3 INDECOPI, Análisis de las funciones del INDECOPI a la luz de las decisiones de sus órganos resolutivos. Protección al consumidor. Colección por el vigésimo aniversario de INDECOPI (Lima: INDECOPI, 2013).

4 La defensa del principio de igualdad constituye un imperativo no solo para los órganos jurisdiccionales, sino también para la justicia administrativa propiamente dicha. Esa materialización de defensa tiene lugar, en modo adicional, a las competencias normativas reguladas de la justicia de la Administración a través del denominado test de igualdad, el mismo que presenta un importante desarrollo en la jurisprudencia constitucional. Edwin Figueroa Gutarra, "El Principio de Igualdad constitucional, límites al trato diferenciado”, Preceptum, 2 (abril de 2015): 29. 


\section{PROTECCIÓN DE LA SALUD}

El artículo 67 del Código de Protección y Defensa del Consumidor, Ley No 29571, literalmente expresa lo siguiente:

\section{Artículo $67^{\circ}$.- PROTECCION DE LA SALUD}

67.1 El proveedor de productos o servicios de salud está en la obligación de proteger la salud del consumidor, conforme a la normativa sobre la materia.

67.2 La prestación de servicios y la comercialización de productos de salud a los consumidores se rigen por las disposiciones establecidas en la Ley $\mathrm{N}^{\circ} 26842$, Ley General de Salud, normas complementarias, modificatorias o las que la sustituyan y en lo que no se oponga por las disposiciones del presente código.

67.3 Los establecimientos de salud y los servicios médicos de apoyo tienen el deber de informar al consumidor, a sus familiares allegados sobre las características del servicio, las condiciones económicas de la prestación y demás términos y condiciones, así como los aspectos esenciales vinculados al acto médico.

67.4 El derecho a la protección de la salud del consumidor es irrenunciable. Sin perjuicio del pleno reconocimiento de estos derechos conforme a la normativa de la materia, los consumidores tienen, de acuerdo al presente Código, entre otros, los siguientes derechos:

a) A que se les brinde información veraz, oportuna y completa sobre las características, condiciones económicas y demás términos y condiciones del producto o servicio brindados.

b) A que se les dé, en términos comprensibles y dentro de las consideraciones de ley, la información completa y continua sobre su proceso, diagnóstico, pronóstico y alternativas de tratamiento, así como sobre las riesgos, contraindicaciones, precauciones y advertencias sobre los productos o servicios brindados.

c) A que se les comunique de forma suficiente, clara y oportuna, veraz y fácilmente accesible, todo lo necesario para que puedan dar su consentimiento informado, previo a la entrega de un producto o la provisión de un servicio.

Previo al análisis del artículo debemos entender quién es el proveedor. La norma define a los proveedores como las personas naturales o jurídicas, de Derecho público o privado, que de manera habitual fabrican, elaboran, manipulan, acondicionan, mezclan, envasan, almacenan, preparan, expenden, suministran productos o prestan servicios de cualquier naturaleza a los consumidores. En forma enunciativa y no limitativa (art. IV del T. P.).

Así mismo define como servicios a "cualquier actividad de prestación de servicios que se ofrecen en el mercado, inclusive las de naturaleza bancaria, financiera, de crédito, de seguros, 
previsionales y los servicios técnicos y profesionales. No están incluidos los servicios que prestan las personas bajo relación de dependencia (art. IV Inc. 5 del T. P). Estos dispositivos resultan bastantes claros para incluir al profesional (en tanto prestador de servicios) como proveedor dentro de una relación de consumo. ${ }^{5}$

El presente artículo está referido a los productos o servicios de salud y a los intereses de los consumidores y usuarios que van a estar protegidos por el Estado, para cuyo efecto se debe garantizar el derecho a la información sobre los bienes y servicios que se encuentran a disposición del consumidor.

La autoridad de salud de nivel nacional es la encargada del control sanitario de los productos farmacéuticos y galénicos, así como del cumplimiento de las disposiciones que se establecen en la ley y su respectivo reglamento; para tal fin, tenemos la Ley General de Salud, Ley 26842, que regula lo mencionado líneas arriba, y reafirma que el órgano rector en materia de salud es el Ministerio de Salud; sin embargo, debemos aclarar que si bien el MINSA es el órgano rector en materia de salud también existen otras entidades que repotencian este campo tan importante en beneficio de la ciudadanía; tal es el caso de la Superintendencia Nacional en Salud, ente clave para el desarrollo del presente artículo.

Hemos mencionado que nuestra Constitución Política del Estado nos señala clara y genéricamente que "todos tienen derecho a la protección de la salud", pero la ley se encarga de mencionar en forma detallada que otros derechos específicos nacen de este derecho fundamental, y como ejemplo tenemos a la Ley General de Salud, Ley 26842 (1997), la ley que modifica la propia Ley General de Salud respecto de la obligación de los establecimientos de salud a dar atención médica en casos de emergencias y partos, Ley 27604 (2001), la Ley que establece los derechos de las personas usuarias de los servicios de salud, Ley 29414 (2009), cuyo reglamento fue aprobado casi recientemente en el 2015, y por ultimo tenemos a la ley que modifica el artículo 11 de la Ley General de Salud y garantiza los derechos de las personas con problemas de salud mental, Ley 29889 (2012); todas estas normas en resumidas cuentas nos mencionan que todos los ciudadanos tenemos derecho a recibir atención médica cuando la necesitemos — más aún si se trata de una emergencia-, a recibir un trato digno, al acceso a la información, a la confidencialidad, a no ser objeto de experimentación para la aplicación de medicamentos, a elegir el centro de atención, al consentimiento informado, etc.

\section{RESPONSABILIDAD POR LA PRESTACION DE SERVICIO DE SALUD}

El artículo $68^{\circ}$ del Código de Protección y Defensa del Consumidor Ley Nº 29571 literalmente expresa lo siguiente:

5 Juan Espinoza Espinoza, Derecho de los Consumidores (Lima: Rodhas, 2006), 253. 
68.1 El establecimiento de salud es responsable por las infracciones al presente Código generadas por el ejercicio negligente, imprudente o imperito de las actividades de los profesionales, de los técnicos o de los auxiliares que se desempeñen en el referido establecimiento, sin perjuicio de las responsabilidades que les correspondan a estos.

68.2 El establecimiento de salud también es responsable por los actos de los profesionales que de manera independiente desarrollen sus actividades empleando la infraestructura o equipos del primero, salvo que el servicio haya sido ofrecido sin utilizar la imagen, reputación o marca del referido establecimiento y esa independencia haya sido informada previa y expresamente al consumidor; sin perjuicio de lo dispuesto en el párrafo 68.1. La responsabilidad del establecimiento de salud conforme a esta norma es solidaria.

El artículo referido protege a los usuarios en cuanto a la responsabilidad por la prestación de servicios de salud brindados por los establecimientos, por cuanto la mayoría de veces son discriminadas y víctimas de una mala atención por parte de las entidades estatales, como el Poder Judicial, ESSALUD, PNP, SUNAT, etc. Es en nombre de esos abusos que cometen las entidades estatales que esta ley protege al consumidor y usuarios. ${ }^{6}$

De una interpretación sistemática de los parágrafos 68.1 y 68.2, el establecimiento de salud es administrativamente responsable solidario con el profesional médico dependiente o aparentemente dependiente. Este artículo repite el error introducido por el Decreto Legislativo 1045. En efecto hay una abierta contradicción con la definición de servicios contenida en el artículo 4 del artículo IV del Título Preliminar, que se aplica a cualquier actividad de prestación de servicios que se ofrece en el mercado inclusive de naturaleza bancaria, financiera, de crédito, de seguros, previsionales y de servicios técnicos y profesionales. No están incluidos los servicios que prestan las personas bajo la relación de dependencia (el énfasis es nuestro). ${ }^{7}$

La obligación por las prestaciones de salud de acuerdo a los medios y en base a lo que espera el consumidor no le asegurará un resultado: no resulta predecible. Se esperará que el servicio sea brindado con la diligencia debida, con la mayor dedicación y utilizando todos los medios requeridos para garantizar el fin deseado. Y la obligación, de acuerdo a los resultados esperados por el consumidor, espera un resultado determinado, previsible, que constituye el fin práctico por el cual se han contratado dichos servicios. La obligación se cumple cuando se ha logrado el resultado. ${ }^{8}$

6 Esteban Carbonell O’Brien, Análisis al Código de Protección y Defensa del Consumidor. Versión actualizada y ampliada (Lima: Juristas Editores EIRL, 2015), 304.

7 Juan Espinoza Espinoza, "Primeras reflexiones a propósito del Código de Protección y Defensa del Consumidor", Ius et Veritas (2010): 168

$8 \quad$ Esteban Carbonell O’Brien, Análisis al Código de Protección..., 304. 


\section{SEGUROS DE SALUD}

El artículo $69^{\circ}$ del Código de Protección y Defensa del Consumidor, Ley N 29571, literalmente expresa lo siguiente:

69.1 Las empresas de seguros están en la obligación de informar clara y destacadamente al consumidor el tipo de póliza y la cobertura de los seguros de salud.

69.2 Las prestaciones de servicios de seguros de salud y el contenido de sus contratos se sujetan a lo dispuesto por la regulación especial emitida por la Superintendencia de Banca, Seguros y Administradoras Privadas de Fondos de Pensiones.

El seguro médico conocido también como seguro de salud o seguro de asistencia sanitaria es un contrato que adquiere el asegurado mediante una póliza, que es un acuerdo con una compañía de seguros. Proporciona al asegurado asistencia médica, hospitalaria o quirúrgica, en caso de enfermedad o accidente, a través de un equipo médico y centros asistenciales, clínicas y hospitales. El contrato de seguro es un contrato por el cual una de las partes (asegurador) se obliga, mediante una prima o dinero que abona el asegurado a la compañía de seguros, a resarcir un daño o cumplir la prestación convenida. Las empresas de seguro tienen la obligación de informar al consumidor el tipo de póliza y la cobertura de los seguros de salud. La prestación de servicio de seguros de salud y el contenido de sus contratos deben observar lo señalado por la regulación especial emitida por la Superintendencia de Banca, Seguros y Administradoras Privadas de Fondos de Pensiones.?

\section{PLANES Y PROGRAMAS OFRECIDOS POR LOS PROVEEDORES DE SERVICIOS DE SALUD}

El artículo $70^{\circ}$ del Código de Protección y Defensa del Consumidor, Ley N ${ }^{\circ} 29571$, literalmente expresa lo siguiente:

Los proveedores de servicios de salud que ofrezcan programas o planes que otorguen beneficios similares o equivalentes a los seguros de salud deben incluir en sus contratos las siguientes condiciones mínimas:

a. Información clara y destacada sobre lo que es materia de servicio. Particularmente debe informarse al consumidor sobre las restricciones en lenguaje de fácil comprensión para personas no versadas en la materia.

b. Entrega bajo cargo de documento escrito en el que consten las condiciones del contrato.

9 Miguel Ramos Bohórquez, Nuevo Código de Protección..., 100. 
c. Entrega bajo cargo de una hoja de resumen en la que consten las exclusiones, coberturas y condiciones relevantes de la póliza en términos comprensibles.

d. Información sobre monto de los beneficios y el precio del programa y plan de salud. La posibilidad de cualquier modificación del precio debe ser advertida de manera destacada al consumidor

e. Información sobre las consecuencias de falta de pago del precio para acceder a los servicios y beneficios estatales.

f. Información sobre el alcance, riesgo y circunstancias exclusivas de la cobertura del programa o plan de salud, las fechas y modalidades de pago del producto o servicio.

g. Información sobre los establecimientos en los cuales puede hacerse valer el servicio contratado y sobre el representante del proveedor encargado de atender los eventos.

h. Información sobre la vigencia del producto o servicio y las condiciones para la renovación del contrato.

El artículo nos menciona que en materia de productos de salud, el Estado promueve el acceso universal a los productos de salud como política pública, con especial incidencia en las poblaciones menos favorecidas económicamente. Dicta y adopta medidas que garanticen el acceso a los medicamentos y dispositivos médicos esenciales, así como se debe disponer de personal idóneo de sugerencias, reclamos o solicitudes de servicios. ${ }^{10}$

El derecho del consumidor y todo movimiento pro consumerista mundial desde hace buen tiempo viene ejerciendo cierta influencia en las normas clásicas del Derecho Civil y en particular sobre la materia contractual, que se ha visto seriamente afectada en sus principios por la evolución económica y social de nuestros tiempos que han debilitado la permanencia e inmutabilidad histórica de las reglas establecidas sobre la base del Derecho romano. En particular, la contratación de consumo como una nueva modalidad tiene gran relación con el Derecho del Consumidor, sobre todo en la preocupación por el llamado contratante débil, que no es una noción individual sino una categoría y una cualidad con lo que se acude al contrato. La contratación de consumo es en cierto modo una contratación derivada de la contratación masiva cuyas expresiones más acabadas son el contrato de adhesión y las cláusulas generales de contratación, y donde la teoría general de las obligaciones ha perdido vigencia en muchos de sus principios basados en una economía agraria y de pequeños comerciantes. ${ }^{11}$

10 Esteban Carbonell O’Brien, Análisis al Código de Protección..., 306.

11 Julio Baltazar Durán Carrión, Derecho del Consumidor. Análisis de su autonomía y nuevas perspectivas de su desarrollo. (Lima: Cultural Cuzco, 2006), 321. 
El negocio jurídico es un acto jurídico, es un acto complejo de dos libertades. En la libertad de celebración, la autonomía privada permite realizar o no realizar el acto y, por lo tanto, optar por la presencia o por la ausencia de determinados efectos de Derecho. En la libertad de estipulación, la autonomía va mucho más allá; ella permite optar por realizar el acto y también seleccionar, más allá de su presencia, el tipo de efectos que producirán. Cuando exista solamente libertad de celebración y de estipulación, surge el negocio jurídico; es la suma de dos libertades. La libertad de celebrar equivale al acto de querer elegir los efectos jurídicos. En el contrato de consumo, el empresario ejerce ambas libertades, mientras que el consumidor solo ejerce la libertad de celebrar el contrato. El consumidor no ejerce la libertad de estipulación porque sencillamente no participa en la reglamentación de los intereses por diferentes razones y técnicas. Una de estas razones es la supremacía psicológica e intelectual del empresario, el cual estaría en ventaja por los conocimientos jurídicos y las experiencias comerciales que inducen al cliente a renunciar a priori a cualquier objeción contra las condiciones generales a él desfavorables. ${ }^{12}$ Por ello, el Estado le exige al proveedor incluir en sus contratos condiciones mínimas de protección al consumidor.

\section{FINANCIAMIENTO DE LOS PROGRAMAS DE SALUD POR UN TERCERO}

El Artículo $71^{\circ}$ del Código de Protección y Defensa del Consumidor, Ley № 29571, literalmente expresa lo siguiente:

Cuando el precio por un programa o plan ofrecido por los proveedores de servicio de salud sea financiado por un tercero distinto a dichos proveedores, se genera una relación de crédito que es independiente del servicio contratado. En estos casos, la prestación de beneficios no está vinculada a la condición del cumplimiento de la obligación crediticia.

Cuando el pago de la cuota de financiamiento sea condición para continuar bajo la cobertura del plan ofrecido, ello debe ser informado expresamente y por escrito a los consumidores de manera previa a la contratación.

En caso de que un tercero, distinto del proveedor, financie un programa o plan ofrecido por los proveedores de servicios de salud, se produce una relación de crédito independiente del servicio que se ha contratado. El crédito básicamente consiste en la entrega de un bien a cambio de la promesa de su restitución o pago futuro. De acuerdo con el texto del Artículo $71^{\circ}$ del Código de Protección y Defensa del Consumidor, la prestación o asistencia de beneficios no está vinculada a la condición de cumplimiento de la obligación crediticia. De otro lado, los proveedores, mediante la concesión de plazos de pago para cubrir el modo de un crédito por unos servicios de salud, hacen posible la existencia de una fuente de financiamiento. ${ }^{13}$

12 Rómulo Morales Hervias, "La definición del contrato del consumo en el Código de Protección y Defensa del Consumidor", Actualidad Jurídica (setiembre de 2010): 202.

13 Miguel Ramos Bohórquez, Nuevo Código de Protección... 


\section{CONDICIONES APLICABLES A LOS SEGUROS DE SALUD, PLANES Y} PROGRAMAS DE SALUD

El artículo $72^{\circ}$ del Código de Protección y Defensa del Consumidor, Ley № 29571, literalmente expresa lo siguiente:

Las empresas de seguros y los proveedores de salud no pueden, mediante la variación unilateral de las condiciones referidas a preexistencias, eliminar las coberturas inicialmente pactadas. Estas disposiciones también se aplican para las renovaciones de los planes de seguros.

El presente artículo está referido a las empresas de seguros y a los proveedores de servicios, los cuales no pueden deshacer las condiciones pactadas en un principio; las condiciones que se pactaron por parte de la empresa o proveedor con el usuario o consumidor van a prevalecer hasta el final del contrato. ${ }^{14}$

\section{LA SUPERINTENDENCIA DE ENTIDADES PRESTADORES DE SALUD Y SU} EVOLUCIÓN A LA SUPERINTENDENCIA NACIONAL DE ASEGURAMIENTO EN SALUD

En el año 1997, con la entrada en vigencia de la Ley de Modernización de la Seguridad Social en Salud, Ley 26790, aparece en el sistema de salud una figura muy importante respecto al modelo de prestaciones de salud que apoyaría en ese entonces al Instituto Peruano de Seguridad Social (IPSS) y pasaría a formar parte del Régimen Contributivo de la Seguridad Social en Salud, nos referimos a las Entidades Prestadoras de Salud (EPS), que se encargarian de prestar servicios con infraestructura propia y de terceros.

Viendo esta situación, era necesario crear un organismo encargado de regular, supervisar y autorizar el funcionamiento de estas empresas, y es así donde nace la Superintendencia de Entidades Prestadoras de Salud (SEPS) como un organismo público descentralizado del sector salud, con personería de Derecho público interno, autonomía funcional, administrativa y financiera.

Con el transcurso de los años y viendo las necesidades de la población, en abril del 2009 se emite la Ley Marco de Aseguramiento Universal en Salud, Ley 29344, como parte de la reforma en el sector salud, estableciendo como pilar el acceso de todo peruano o residente nacionalizado a un plan de aseguramiento en salud, el cual se establecería dentro de los regímenes de financiamiento ${ }^{15}$ en salud que la propia ley clasificaría como régimen contributivo, semicontributivo y subsidiado.

14 Esteban Carbonell O’Brien, Análisis al Código de Protección..., 308.

15 Artículo 29 del Texto Único Ordenado de la Ley 29344, respecto a los regímenes de financiamiento en salud. 
En razón de ello es que se repotencia la figura de la Superintendencia de Entidades Prestadoras de Salud y se crea la Superintendencia Nacional de Aseguramiento en Salud (SUNASA), encargada de supervisar y fiscalizar las relaciones entre Instituciones Administradoras de Fondos de Aseguramiento en Salud (IAFAS), Instituciones Prestadoras de Servicios de Salud (IPRESS) frente a la población asegurada.

Las clases de Instituciones Administradoras de Fondos de Aseguramiento en Salud ${ }^{16}$ son:

a) Seguro Integral de Salud.

b) Seguro Social de Salud (ESSALUD), excluyendo la cobertura de prestaciones económicas y sociales.

c) Fondo Intangible Solidario de Salud (FISSAL).

d) Fondos de Aseguramiento en Salud de las Fuerzas Armadas y de la Policía Nacional del Perú.

e) Entidades Prestadoras de Salud (EPS).

f) Empresas de seguros contempladas en los numerales 1, 2 y 3 del inciso d) del Artículo 16 de la Ley $26702,{ }^{17}$ que oferten cobertura de riesgos de salud de modo exclusivo o en adición a otro tipo de coberturas.

i) Asociaciones de Fondos Regionales y Provinciales contra Accidentes de Tránsito (AFOCAT).

j) Entidades de salud que ofrecen servicios de salud prepagadas.

1) Autoseguros y fondos de salud que gestionen fondos para la cobertura de salud de modo exclusivo o en adición a otro tipo de coberturas.

o) Otras modalidades de aseguramiento público, privados o mixtos distintas a las señaladas anteriormente.

16 Artículo 6 del Decreto Legislativo No 1158, que dispone medidas destinadas al fortalecimiento y cambio de denominación de la Superintendencia Nacional de Aseguramiento en Salud, en concordancia con el artículo 7 del Texto Único Ordenado de la Ley Marco de Aseguramiento Universal en Salud, Decreto Supremo No 020-2014-SA.

17 Ley General del Sistema Financiero y de Seguros y Orgánica de la Superintendencia de Banca y Seguros, en lo concerniente a empresas de seguros:

1.- Empresa que opera en un solo ramo (de riesgos generales o de vida),

2.- Empresa que opera en ambos ramos (de riesgos generales y de vida),

3.- Empresa de seguros y de reaseguros. 
Entonces, de acuerdo con lo establecido en la norma, las IAFAS son aquellas instituciones o empresas que financian las atenciones médico-quirúrgicas de sus asegurados, con fondos que provienen de una contribución por parte del propio afiliado o por parte del Estado a manera de subsidio.

Tenemos como IPRESS a los siguientes:

a) Seguro Social de Salud (ESSALUD).

b) Los centros asistenciales del Ministerio de Salud.

c) Las clínicas privadas.

d) Los hospitales de las Fuerzas Armadas y Policiales.

f) Las Entidades Prestadoras de Salud (EPS).

h) Otros centros que prestan servicios de salud.

Entendemos por Instituciones Prestadoras de Servicios de Salud a los establecimientos de salud y servicios médicos de apoyo, públicos, privados o mixtos, creados o por crearse, que realizan atención de salud con fines de prevención, promoción, diagnóstico, tratamiento y/o rehabilitación; así como aquellos servicios complementario o auxiliares de la atención médica, que tienen por finalidad coadyuvar en la prevención, promoción, diagnóstico, tratamiento y/o rehabilitación de la salud. ${ }^{18}$

\section{LA DEFENSORÍA DE LA SALUD Y TRANSPARENCIA COMO ORGANISMO DEL MINISTERIO DE SALUD}

La Defensoría de la Salud y Transparencia (dependiente del Ministerio de Salud) era responsable de la protección y defensa de los derechos en salud hasta 120 días (Cuarta disposición complementaria final) posteriores a la publicación del D.L. No 1158, que fue el 06 de diciembre de 2013, el cual tenía como función intervenir de oficio o a solicitud de parte en los hechos o actos que vulneren o pudieran vulnerar el derecho a la salud de la persona, la familia y la comunidad, así como en los hechos que atenten contra la ética y transparencia en la gestión administrativa del Ministerio de Salud.

Paralelamente, la SUNASA intervenía frente a la vulneración o situaciones que podían configurarse como tal, sobre la población asegurada indistintamente de la institución administradora de fondos de aseguramiento a la que pertenezca. A ello también debemos mencionar que otras entidades como la Defensoría del Pueblo y el INDECOPI se

\footnotetext{
18 Artículo 7 del Decreto Legislativo No 1158, respecto a las Instituciones Prestadoras de Servicios de Salud - IPRESS
} 
encargaban de la protección de los derechos de los usuarios de los servicios de salud, sean o no asegurados (hasta el 06 de diciembre de 2013, publicación del D.L. No 1158), pero lamentablemente encontrar una solución frente a estos problemas tardaba mucho debido a la carga procedimental.

Entonces, era evidente la existencia de una multiplicidad de esfuerzos y recursos, diversos agentes para la protección de los derechos en salud, falta de regulación y supervisión de los procedimientos para la atención de reclamos y quejas a cargo de las instancias de atención a nivel local, regional y nacional, agudizando más este déficit el hecho de que el propio ciudadano no cuente con toda la información sobre sus derechos.

Por ello, contar con una institución que asegure la presencia de órganos de protección de derechos a nivel nacional era crucial, puesto que posibilita que un amplio sector de la población que anteriormente no contaba con canales adecuados de participación actualmente pueda conocer sus derechos en salud y acceder a mecanismos que le permitan exigir su cumplimiento. Mediante la cuarta disposición complementaria final del Decreto Legislativo No 1158, SUSALUD asume el acervo documentario, los procedimientos y funciones de la Defensoría de la Salud y Transparencia del Ministerio de Salud.

\section{CAMBIO DE DENOMINACIÓN, FORTALECIMIENTO Y AMPLIACIÓN DE COMPETENCIADELASUPERINTENDENCIANACIONALDEASEGURAMIENTO EN SALUD}

La SUNASA estaba encargada de supervisar, fiscalizar y sancionar las relaciones entre entidades prestadoras y financiadoras de servicios de salud y sus usuarios, es decir, su intervención se limitaba a las relaciones entre entidad aseguradora y asegurado, dejando la protección e intervención de los ciudadanos no asegurados al MINSA mediante sus órganos competentes y el INDECOPI.

El 06 de diciembre de 2013 se publicó en el Diario Oficial El Peruano el Decreto Legislativo No 1158, que dispone medidas destinadas al fortalecimiento y cambio de denominación de la Superintendencia Nacional de Aseguramiento en Salud (SUNASA) a Superintendencia Nacional de Salud (SUSALUD).

Muchos consideran que solo se trata de un cambio denominativo; sin embargo, es mucho más que eso puesto que se amplía la competencia de este organismo. Es necesario indicar que a raíz de la publicación del Decreto Legislativo No 1158, la función fiscalizadora y sancionadora de la Superintendencia Nacional el Salud no solo se circunscribe a una relación de aseguramiento. 
Entre los puntos más importantes que motivaron ${ }^{19}$ el cambio, fortalecimiento y ampliación de la Superintendencia tenemos los siguientes:

a) El sistema Nacional de Protección de los Derechos de los usuarios de los servicios de salud se vincula directamente con la labor de la Superintendencia Nacional de Aseguramiento en Salud, por lo que resulta necesario que sea una sola entidad la que resguarde, garantice y promueva los derechos de las personas al acceso a los servicios de salud, velando por prestaciones con calidad, oportunidad y aceptabilidad, con independencia de la fuente de financiamiento, así como los que correspondan en su relación de consumo con las IAFAS o IPRESS. Así mismo, debe contar con funciones que le permitan promover y vigilar la participación ciudadana, construyendo para estos efectos los canales, redes de diálogo y de protección de derechos con las organizaciones de la sociedad civil y entidades del sector Salud.

b) Se requiere contar con un único sistema que contenga la información de los establecimientos de salud y servicios médicos de apoyo, el mismo que deberá ser materia de consulta y uso por parte de los gobiernos regionales u otros agentes debidamente certificados para los procesos de registro, categorización y acreditación.

c) Fortalecer el rol rector del Ministerio de Salud contando con una entidad que asuma plenamente la responsabilidad de ejercer las facultades para administrar, fiscalizar y sancionar los procesos de categorización a nivel nacional, promoviendo la especialización de las funciones antes mencionadas y la complementariedad con la autoridad sanitaria regional, sin menoscabo de la función reguladora del MINSA.

d) La Superintendencia, en su condición de organismo especializado en materia de salud, debe otorgar un sistema de protección equivalente al que brinda el INDECOPI, motivo por el cual corresponde su fortalecimiento, incorporando la posibilidad de dictar medidas correctivas y de carácter provisional. Así mismo requiere contar con una instancia de resolución colegiada cuyas decisiones generen precedentes vinculantes y sean revisadas únicamente a partir de la Sala Contencioso Administrativa de la Corte Superior respectiva, de acuerdo al Artículo Único de la Ley 27709, que a su vez modificó la Ley 27584, Ley que regula el Proceso Contencioso Administrativo, para lo cual se requiere modificar la norma en cuestión incorporando el Tribunal de la Superintendencia.

19 Exposición de motivos por parte del Poder Ejecutivo, para la elaboración de la Ley que dispone el fortalecimiento de la Superintendencia Nacional de Aseguramiento en Salud y su cambio de denominación a Superintendencia Nacional de Salud, pp. 6, 7, 8 . 
Como apreciamos, la necesidad de ampliar la competencia de la Superintendencia estuvo relacionada con fortalecer el rol protector de derechos y libre acceso de los ciudadanos a las prestaciones de salud, tal como señala el artículo 11 de nuestra Constitución Política; si bien el Ministerio de Salud se encarga de regular el sistema de salud en el país, SUSALUD se encarga de fiscalizar, supervisar y sancionar las relaciones entre entidades que financian y prestan servicios de salud a la ciudadanía, garantizando el correcto desarrollo de las políticas de Estado bajo el principio de pro usuario.

El Decreto Legislativo No 1158 (2013), en su Artículo 8, Numeral 1, sobre las funciones generales, nos dice:

Son funciones de la Superintendencia Nacional de Salud el promover, proteger y defender los derechos de las personas al acceso a los servicios de salud, supervisando que las prestaciones sean otorgadas con calidad, oportunidad, disponibilidad y aceptabilidad, con independencia de quien las financie, así como los que correspondan en su relación de consumo con las IAFAS o IPRESS, incluyendo aquellas previas y derivadas de dicha relación (...).

La norma confirma que SUSALUD tiene como objetivo principal la promoción y protección de derechos de los usuarios en los servicios de salud, sin distinción alguna, y parte de la promoción es dar a conocer a la ciudadanía sobre sus derechos en materia de salud para fortalecer y equilibrar este binomio relacional entre entidad y usuario.

De igual modo, es importante precisar que SUSALUD cuenta con potestad sancionadora sobre toda acción u omisión que afecte el derecho a la vida, la salud, la información de las personas usuarias de los servicios de salud y la cobertura para su aseguramiento, también respecto a los estándares de acceso, calidad, oportunidad, disponibilidad y aceptabilidad con que dichas prestaciones sean otorgadas; y para la ejecución de los actos administrativos firmes se podrá ejercer los medios de ejecución forzosa previstos en la norma vigente, respetando los principios de proporcionalidad y razonabilidad de acuerdo a lo señalado en el Ley de Procedimiento Administrativo General, Ley 27444.

Para la solución de controversias, la Superintendencia cuenta con el Centro de Conciliación y Arbitraje (CECONAR), competente para conocer y resolver las controversias que surjan entre los agentes que forman parte del Sistema Nacional de Salud, a través del establecimiento de mecanismos de solución de conflictos; y por ultimo cuenta con el Tribunal de la Superintendencia, que es competente para conocer y resolver en última instancia administrativa los procedimientos y materias sometidas a su consideración, cuyas resoluciones podrán ser impugnadas únicamente ante la Corte Superior a través de la acción contenciosa administrativa, tal como lo indica el último párrafo del Artículo 24 del Decreto Legislativo $\mathrm{N}^{\circ} 1158$. 


\section{ARTICULACIÓN DE COMPETENCIA CON EL INSTITUTO NACIONAL DE DEFENSA DE LA COMPETENCIA Y DE LA PROTECCIÓN DE LA PROPIEDAD INTELECTUAL (INDECOPI)}

El Código de Protección y Defensa al Consumidor, Ley 29571, en su artículo 105, señala que el Instituto Nacional de Defensa de la Competencia y de la Protección de la Propiedad Intelectual (INDECOPI) es la autoridad con competencia primaria y de alcance nacional para conocer las presuntas infracciones a las disposiciones contenidas en el precitado Código, así como para imponer las sanciones y medidas correctivas que en dicha norma se establecen.

Esta competencia solo puede dejarse sin efecto o negarse cuando haya sido asignada a favor de otro organismo u entidad por norma expresa con rango de ley, y además, se cuente con un sistema de protección equivalente en capacidades, lo que significa tener la capacidad de imponer medidas correctivas, dictar medidas de ejecución forzosa, contar con un órgano colegiado para la resolución de la vía administrativa, y poder tener una vía debidamente articulada, en base al principio de especialidad, en el proceso contencioso administrativo.

Entonces, al crearse la Superintendencia Nacional de Aseguramiento en Salud (SUNASA) de acuerdo a la Ley Marco de Aseguramiento Universal en Salud, Ley 29344, se estaría cumpliendo con la condición inicial de contar con un organismo especializado en la solución de controversias en materia de salud; sin embargo, desde la posición del Código de Protección y Defensa al Consumidor, esto no era suficiente puesto que dicha protección debía ser garantizada a todos los ciudadanos inmersos en la materia; en razón de ello es que se crea la Superintendencia Nacional de Salud (SUSALUD), completándose este rol de protección de derechos de los usuarios a los servicios de salud sean o no asegurados, canalizado mediante un ente especializado.

TRANSFERENCIA DE FUNCIONES DE INDECOPI A SUSALUD, EN EL MARCO DEL DECRETO LEGISLATIVO No 1158

El pasado 13 de agosto de 2015 se publicó en el Diario Oficial El Peruano el Decreto Supremo No 026-2015-SA que aprueba el Reglamento del Procedimiento de Transferencia de Funciones del Instituto Nacional de Defensa de la Competencia y de la Protección de la Propiedad Intelectual (INDECOPI) a la Superintendencia Nacional de Salud (SUSALUD), en el marco del Decreto Legislativo No 1158; esta norma responde a la iniciativa de fortalecer y ampliar las facultades de la Superintendencia en razón de los motivos ya señalados anteriormente, para lo cual mencionaremos las principales funciones atribuidas a SUSALUD.

Entre las principales funciones transferidas tenemos:

a) La promoción, protección y defensa de derechos de las personas al acceso a los servicios de salud. 
b) Conocer con competencia primaria y alcance nacional las presuntas infracciones a las disposiciones relativas a la protección de los derechos de los usuarios en su relación de consumo con la IPRESS y/o IAFAS.

d) La identificación de cláusulas abusivas en los contratos o convenios que suscriben las IAFAS con los asegurados o entidades que los representen, según las disposiciones aplicables de la Ley 29571, ${ }^{20}$ con excepción de las pólizas de seguros de las empresas de seguros bajo el control de la Superintendencia de Banca, Seguros y AFP.

e) Velar por el cumplimiento del Código de Protección y Defensa del Consumidor, normas complementarias y conexas, en materia de protección del derecho de los usuarios de los servicios de salud, por la falta de idoneidad de los servicios ofrecidos por las IAFAS, IPRESS y UGIPRESS, ejerciendo su potestad sancionadora.

f) Supervisar que las IPRESS cumplan con lo dispuesto en los artículos 67 y 68 de la Ley $29571 .{ }^{21}$

i) Supervisar el otorgamiento de prestaciones y cobertura para los consumidores del Seguro Complementario de Trabajo de Riesgo (SCTR), la cobertura del Seguro Obligatorio de Accidentes de Tránsito (SOAT) y de las Asociaciones de Fondos Regionales y Provinciales Contra Accidentes de Tránsito (AFOCAT) respetando los límites de Ley. ${ }^{22}$

A modo de síntesis podemos indicar que la Superintendencia Nacional de Salud es la competente para intervenir frente a conflictos o vulneración de derechos de los consumidores de un plan de aseguramiento específico, teniendo en cuenta que los servicios que brindan las IAFAS e IPRESS no deben ser menores al plan esencial de aseguramiento en salud (PEAS), que es el listado priorizado de diagnósticos e intervenciones médicas regulado por la Ley Marco de Aseguramiento Universal en Salud.

Sin embargo, cuando nos referimos a las pólizas de seguros, o prestaciones económicas como el caso de pensiones de invalidez, gastos de sepelio y otros semejantes, las entidades competentes para intervenir son el INDECOPI y la Superintendencia de Banca, Seguros y AFP.

20 Código de Protección y Defensa del Consumidor, Artículo 50, respecto a cláusulas abusivas de ineficacia absoluta, y Artículo 51 respecto a cláusulas abusivas de ineficacia relativa.

21 Código de Protección y Defensa del Consumidor, Artículo 67, respecto a protección de la salud y Artículo 68, respecto a responsabilidad por la prestación de servicios de salud.

22 Los Artículos 7 y 8 del Decreto Supremo No 026-2015-SA, respecto a las competencias sobre el Seguro Complementario de Trabajo de Riesgo y Empresas de Seguros, SOAT y AFOCAT, nos dicen que la Superintendencia de Banca Seguros y AFP (SBS) e INDECOPI mantienen las facultades para actuar en la vía administrativa en materia referida a las prestaciones económicas, como es el caso de las pensiones de sobrevivencia, pensiones de invalidez, gastos de sepelio u otros. 


\section{LAS INFRACCIONESY SANCIONES IMPUESTAS POR LA SUPERINTENDENCIA NACIONAL DE SALUD}

El 06 de noviembre de 2014, mediante Decreto Supremo No 031-2014-SA, se publicó en el Diario Oficial El Peruano el Reglamento de Infracción y Sanciones de la Superintendencia Nacional de Salud, cuya vacatio legis fueron 30 días.

En la mencionada norma se señala que el inicio del procedimiento administrativo sancionador podrá ser de oficio cuando se trate de una presunta comisión de infracciones y también a raíz de una queja interpuesta ante SUSALUD.

Entre los tipos de sanciones tenemos:

1. Amonestación escrita, que se refiere a una llamada de atención a la IAFAS y/o IPRESS, mediante documento. Es la sanción más leve.

2. Multa hasta un monto máximo de quinientas (500) UIT, dentro de los parámetros establecidos en el artículo 21 del reglamento. ${ }^{23}$ Es la sanción pecuniaria sin carácter indemnizatorio o resarcitorio para los afectados, que busca morigerar la actuación errada de la IAFAS sancionada, la cual deberá ser cancelada dentro de los quince (15) días hábiles siguiente de hacer quedado firme la resolución de sanción.

3. Suspensión de la autorización de funcionamiento para IAFAS, hasta por un plazo máximo de seis (6) meses. Significa la prohibición temporal para efectuar nuevas afiliaciones o participar en los procesos de elección de las EPS, e incluso la situación de las empresas que operan en un solo ramo, en ambos ramos y empresas de seguros y reaseguros, inmersas en el procedimiento sancionador, será puesta en conocimiento de la Superintendencia de Banca, Seguros y AFP, para los fines de su competencia.

4. Restricción de uno o más servicios de las IPRESS, hasta por un plazo máximo de seis (6) meses. Es la prohibición para brindar prestaciones de salud, servicios médicos de apoyo o servicios auxiliares o complementarios en los centros de servicios públicos, privados o mixtos, registrados o no en SUSALUD.

5. Cierre temporal de IPRESS, hasta por un plazo máximo de seis (6) meses. Significa la prohibición temporal de brindar prestaciones de servicios de salud y para la habilitación de la IPRESS. Finalizado el periodo de suspensión, restricción o cierre temporal, se deberán acreditar las observaciones que motivaron la sanción, con lo que la Intendencia de Normas y Autorizaciones (INA) procederá a la habilitación de la Autorización de Funcionamiento o de Registro, según corresponda.

23 El Artículo 21 del Decreto Supremo No 031-2014-SA, Reglamento de Infracciones y Sanciones de la Superintendencia Nacional de Salud, nos indica sobre el rango de sanciones para IAFAS, IPRESS y UGIPRESS. 
6. Revocación de la autorización de funcionamiento para IAFAS. Acarrea la pérdida definitiva del derecho de las IAFAS a desarrollar, por sí misma o a través de terceros, las actividades de captación, recepción y/o gestión de fondos para la cobertura de las atenciones de salud o la oferta de cobertura de riesgos de salud, bajo cualquier modalidad. Las IAFAS cuyo único objeto social o finalidad sea la de brindar servicios de cobertura de riesgos de salud y/o de captación y gestión de fondos para el aseguramiento de las prestaciones de salud deberán iniciar su proceso de disolución y liquidación de propia iniciativa o a solicitud de los acreedores, conforme a los procedimientos establecidos en las normas de la materia, que para el caso es el procedimiento concursal preventivo.

7. Cierre definitivo de IPRESS. No podrá realizar atenciones de salud con fines de prevención, promoción, diagnóstico, tratamiento y/o rehabilitación, ni de servicios complementarios o auxiliares de la atención médica que tengan por finalidad coadyuvar en la prevención, promoción, diagnóstico, tratamiento y/o rehabilitación de la salud. Dicha sanción será comunicada por la Superintendencia Adjunta de Regulación y Fiscalización (SAREFIS) a las IAFAS que mantengan vínculo con la IPRESS sancionada, a fin de que adopten las medidas necesarias para garantizar a sus afiliados el acceso, calidad y oportunidad en la prestación de los servicios de salud, sin perjuicio de su derecho a repetir contra la IPRESS sancionada por el incumplimiento de la oferta.

En el reglamento se aprecian las medidas de carácter provisional y correctivo, los criterios para la aplicación de sanciones y el régimen de subsanación de infracciones. Cabe mencionar que las presentes sanciones administrativas previstas en el reglamento se aplican sin perjuicio de la responsabilidad civil o penal que pudiera derivarse de los actos u omisiones constitutivos de infracción, los cuales se regulan de acuerdo a lo previsto en la legislación de la materia.

\section{REFLEXIÓN DE LOS AUTORES}

Hablar de protección de derechos y lograr que la ciudadanía entienda esta figura jurídica es muy complejo cuando el mismo ciudadano desconoce sus derechos. Casi siempre acudimos a una entidad a presentar una queja o reclamo cuando nos vemos vulnerados en nuestra economía (pago de una multa o cuando nos cobran más de lo debido), lo que evidentemente es preocupante; sin embargo, la protección del consumidor como usuario de los servicios de salud aborda muchos aspectos y debería ser materia de reflexión de las dos partes involucradas, es decir, de las entidades que prestan servicios de salud y de los propios usuarios.

La Superintendencia Nacional en Salud es el órgano competente para la promoción y protección de derechos de los usuarios de los servicios de salud tal como señala el Decreto Legislativo No 1158. A todo ello debemos mencionar que la mayoría de usuarios han interiorizado y aceptado "el falso estándar de buen trato" por parte del servidor público o privado, el cual se limita a la explicación del malestar, indicación de la medicación o receta, 
los cuidados que debe tener en cuenta el paciente, etc. Pero olvidan que esta relación o binomio relacional entre usuario y prestador de servicio necesita la calidad humana basada en la cordialidad, amabilidad, respeto y prestación oportuna, tal como lo señala la Ley 29414, Ley que establece los Derechos de las Personas Usuarias de los Servicios de Salud, publicada en el 2009 y reglamentada en el 2015, la cual se considera una extensión de la Ley General de Salud, que también menciona los derechos de los usuarios en los servicios de salud.

\section{CONCLUSIONES}

1. En el año 2015, a partir de la emisión del Decreto Supremo No 026-2015-SA, el cual responde a la ampliación de facultades de la antigua SUNASA en el marco del Decreto Legislativo No 1158 dado en el año 2013, la facultad para proteger al consumidor en los servicios de salud es transferida de INDECOPI a la hoy llamada Superintendencia Nacional de Salud (SUSALUD), con el propósito de canalizar la gran demanda de quejas y reclamos a este organismo especializado en protección de derechos de los usuarios de salud, y además con la finalidad de reafirmar la promoción y defensa de los derechos de la ciudadanía en materia de salud sea o no asegurada, garantizando la intervención oportuna de la Superintendencia Nacional de Salud en razón a su facultad fiscalizadora y sancionadora.

2. El ciudadano de a pie debe tener en cuenta que frente a la necesidad de hacer valer sus derechos en materia de salud, ya sea mediante una queja, reclamo, solicitud o sugerencia, tiene a la Superintendencia Nacional en Salud, la que se encargará de hacer las investigaciones correspondientes para luego aplicar medidas correctivas o sanciones, de corresponder.

\section{REFERENCIAS}

- Bullar Gonzales, Alfredo. "Código de Consumo: el discreto encanto del socialismo". El Comercio, 10 de abril de 2010.

- Carbonell O’Brien, Esteban. Análisis al Código de Protección y Defensa del Consumidor. Versión actualizada y ampliada. Lima: Juristas Editores EIRL, 2015.

- Durán Carrión, Julio Baltazar. Derecho del Consumidor. Análisis de su autonomía y nuevas perspectivas de su desarrollo. Lima: Cultural Cuzco, 2006.

- Espinoza Espinoza, Juan. Derecho de los Consumidores. Lima: Rodhas, 2006.

"Primeras reflexiones a propósito del Código de Protección y Defensa del Consumidor". Ius et Veritas (2010): 168. 
- Figueroa Gutarra, Edwin. "El Principio de Igualdad constitucional, límites al trato diferenciado". Preceptum, 2 (abril de 2015).

- INDECOPI. Análisis de |las funciones del INDECOPI a la luz de las decisiones de sus órganos resolutivos. Protección al consumidor. Colección por el vigésimo aniversario de INDECOPI. Lima: INDECOPI, 2013.

- Morales Hervias, Rómulo. "La definición del contrato del consumo en el Código de Protección y Defensa del Consumidor". Actualidad Jurídica (setiembre de 2010).

- Ramos Bohórquez, Miguel. Nuevo Código de Protección y Defensa del Consumidor. Ley $N^{\circ}$ 29571. Edición 2014.

Recibido: $12 / 04 / 2016$

Aceptado: 20/05/2016 


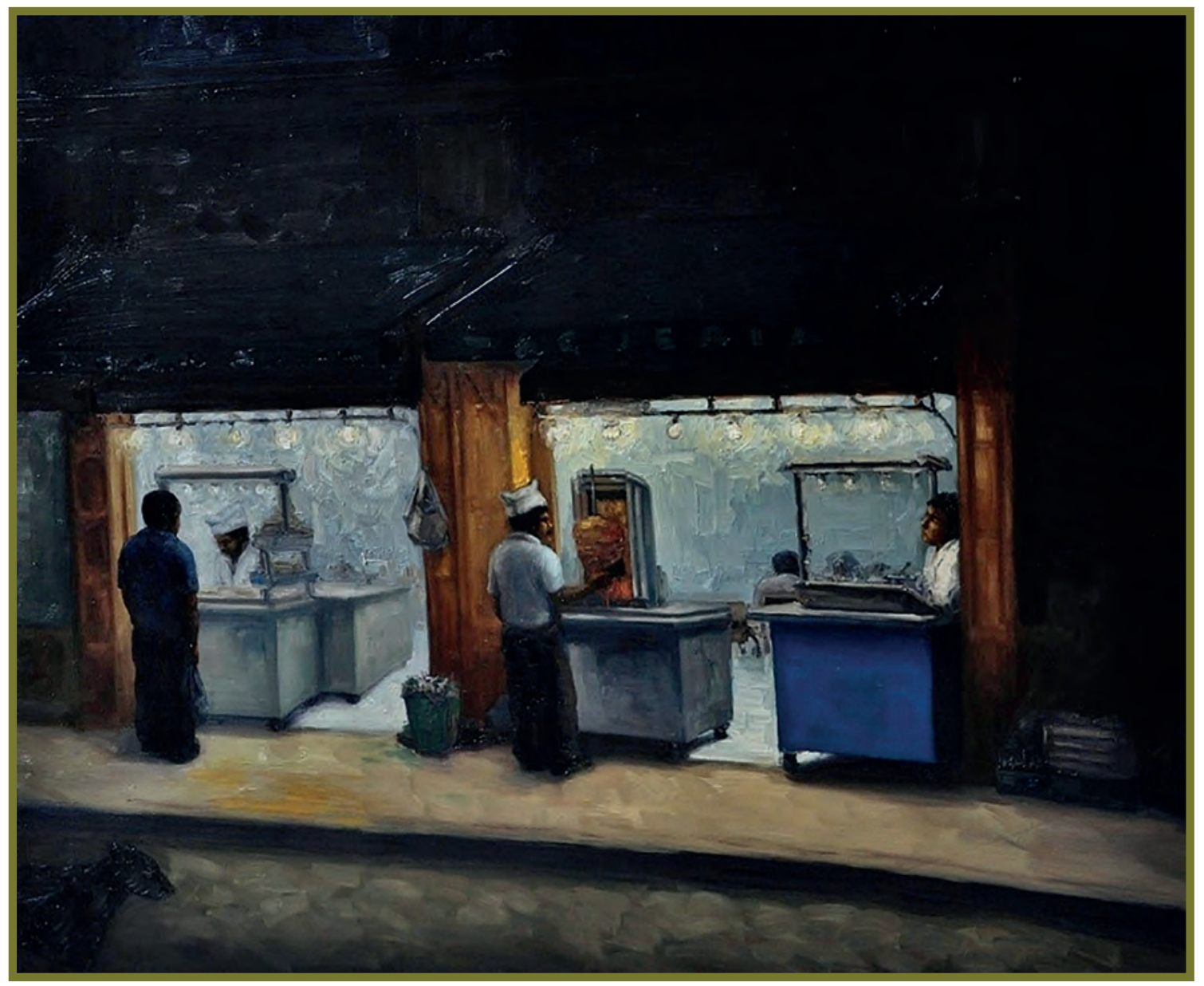

Vendedores de tacos $(90 \mathrm{~cm}$ x $120 \mathrm{~cm})$. Diego Alcalde Taboada. 